\title{
The dangerous battles over sex and gender
}

Book review of Testosterone Rex: Unmaking the Myths of our Gendered Minds by Cordelia Fine, Icon Books, 2017. £14.99, hbk (265 pp.) ISBN 978-178578-161-2.

Gillian R. Brown (grb4@st-andrews.ac.uk), School of Psychology \& Neuroscience, University of St Andrews, St Andrews, UK

Published in Trends in Ecology and Evolution https://doi.org/10.1016/j.tree.2017.10.003

Within the academic community, the issue of gender equality is gaining unprecedented amounts of attention, with emotive discussions about discrimination and bias in the workplace proliferating on social media platforms and in established print outlets. Initiatives that reward universities for engaging with this agenda, such as the UK's Athena SWAN scheme, have started to spread out across the globe, while funding bodies increasingly monitor gender balance amongst their grant awardees. Within this changing landscape, academics that are pushing against stereotypical views of the psychological traits of women and men are likely to find an increasingly receptive audience for their ideas. Entering this arena, Cordelia Fine, in her new book, Testosterone Rex, attacks the idea that the eponymous hormone is responsible for imbuing the males of our species with specific strengths and attributes that could alternatively have arisen through socialisation and cultural processes. Building on her well-received previous book, Delusions of Gender [1], the author's underlying agenda is clear, namely to argue that if we fall into the trap of believing that testosterone is the kingpin that determines sex differences in behaviour and cognition, gender equality is likely to be impossible to reach.

Testosterone Rex is an engagingly-written book, often witty and frequently acerbic, that aims to dismantle the evidence that testosterone is responsible for producing 'male' brains, as opposed to 'female' brains, and that testosterone thereby acts 'as a powerful, potent, polarizing developmental force' (page 177). Although neuroscientists have long known that testosterone can act upon neural cells, Fine is unwilling to accept overly simplistic accounts that link early testosterone exposure with sex differences in the brain and behaviour. The author is particularly adept at deconstructing arguments that rely on unsubstantiated accounts of how females and males in our species have evolved. To biologists, some of these arguments will be familiar and relatively uncontroversial. For instance, when discussing Bateman's principals, which have provided insights into how sex differences in competitiveness and choosiness in mating scenarios might have evolved, Fine points to recent theoretical research showing that multiple factors, other than just levels of parental care, influence 
mating strategies. The complexities of human mating strategies are therefore unlikely to be explained by evolutionary accounts that perpetuate stereotypical accounts of the sexes [2].

Fine's argument is most convincing when the target of her wrath is an extreme version of evolutionary psychology that relies on 'Just So' storytelling and unsupported assumptions about how evolutionary selection pressures acted on our human ancestors. In places, Fine could be accused of selectively presenting only the evidence that supports her argument, while counter-evidence is often relegated to footnotes or simply dismissed. As such, the book should be viewed as an opinion piece, rather than an overview of the whole field. However, moving away from stereotypical views that are only superficially supported, or that rely on scientific evidence that is flawed either in terms of the quality of the empirical evidence or the underlying theoretical assumptions, is crucial in terms of enhancing the quality of our research base, and a clearer picture could reduce gender-based prejudices and barriers within our societies. Fine is correct in pointing out that our expectations about how women and men have evolved have real-world implications for how we think about current sex roles and their malleability, and the author's unwavering position provides a strong counter-point to extreme nativist views of sex differences that often abound within the popular science literature.

That said, one disappointing aspect of this book is the lack of coverage of the large body of experimental studies showing that testosterone exposure in early life does impact upon the development of the brain and behaviour in non-human animals. With apparent reluctance, Fine briefly admits that such research indicates that testosterone has cellular actions that can influence neurodevelopmental processes. Notably, Fine uses the abbreviation $\mathrm{T}$, rather than the word testosterone, in the short section that refers to the physiological effects of this hormone on neural functioning, which suggests that the author is genuinely conflicted about how to incorporate this experimental evidence into her world view. Fine also acknowledges that studying the role of hormones in these neurodevelopmental processes is likely to be important for understanding why men and women are differentially susceptible to certain mental health disorders. Yet Fine's aversion to the idea that testosterone plays a significant role in human behavioural development remains unflinching. The omission of scientifically justified, carefully controlled experiments on hormones and brain development will likely be met with frustration by many within the research community.

Testosterone Rex achieves its goal of deconstructing explanations of sex differences in human behaviour and psychological traits that are overly reliant on unsubstantiated evidence and weak assumptions. However, Fine's stand-point potentially leaves scientists occupying in the middle-ground between extreme nativist views of sex differences and those extoling the importance of social processes at risk of being struck by the violently swinging pendulum of this nature-nurture debate. Acknowledging that 
hormones can influence neurodevelopmental trajectories should not be viewed as antithetical to the idea that human sex roles are largely socially prescribed. Hormones act in a dynamically changing, bidirectional way to mediate both genetic and environmental influences on phenotypes, thereby conferring flexibility onto developmental processes. Hormones can thus contribute to the constructive development of individuals throughout their lifespans and beyond, including via trans-generational epigenetic mechanisms, with potential implications for evolutionary dynamics. This more complex account of hormonal actions, which is consistent with an extended view of evolutionary theory [3], now deserves to be brought to the fore. Otherwise, embittered battles over sex differences could continue raging for many more decades to come.

\section{References}

1. Fine, C. (2010) Delusions of Gender: the Real Science Behind Sex Differences, Icon Books

2. Brown, G.R. et al. (2009) Bateman's principles and human sex roles. Trends Ecol. Evol. 24, 297-304

3. Laland, K.N. et al. (2015) The extended evolutionary synthesis: its structure, assumptions and predictions. Proc. R. Soc. B 282, 20151019. 